\title{
Uniform Blow-up Rate for Nonlocal Diffusion-like Equations with Nonlocal Nonlinear Source
}

\author{
Jiashan ZHENG
}

Ludong University

(Communicated by K. Matsuzaki)

\begin{abstract}
We present new blow-up results for nonlocal reaction-diffusion equations with nonlocal nonlinearities. The nonlocal source terms we consider are of several types, and are relevant to various models in physics and engineering. They may involve an integral of an unknown function, either in space, in time, or both in space and time, or they may depend on localized values of the solution. We first show the existence and uniqueness of the solution to problem relying on contraction mapping fixed point theorem. Then, the comparison principles for problem are established through a standard method. Finally, for the radially symmetric and non-increasing initial data, we give a complete classification in terms of global and single point blow-up according to the parameters. Moreover, the blow-up rates are also determined in each case.
\end{abstract}

\section{Introduction}

Our goal is to study the blow-up rate of the nonlocal heat equation with the nonlocal nonlinear reaction terms, namely,

$$
\left\{\begin{array}{l}
u_{t}=\int_{\mathbf{R}^{N}} J(x-y)(u(y, t)-u(x, t)) d y+a(x)\left(\int_{\Omega}|u(x, t)|^{r} d x\right)^{\frac{p}{r}}, \quad x \in \Omega, t>0, \\
u(x, t)=0, \quad x \notin \Omega, \quad t \geq 0, \\
u(x, 0)=u_{0}(x), \quad x \in \Omega,
\end{array}\right.
$$

where $\Omega$ is a unitary ball, $J: \mathbf{R}^{N} \rightarrow \mathbf{R}$ is a nonnegative, smooth, radially symmetric function with $\int_{\mathbf{R}^{N}} J(z) d z=1$ and supported in the unit ball, $p>1, r \geq 1, a(x), u_{0}(x) \in C^{2}(\Omega)$; $a(x), u_{0}(x)>0$ in $\Omega$ and $a(x)=u_{0}(x)=0$ on $\partial \Omega$.

Nonlocal problems related to (1.1) have been recently widely used to model diffusion processes. Since the Laplacian operator in the reaction diffusion system is not sufficiently accurate in modeling the spatial diffusion of the individuals in some cases (see [17, 22]), especially in many biological areas, such as the embryological development cases. One way

Received February 28, 2015; revised July 10, 2015

2010 Mathematics Subject Classification: 35K57, 35B33, 35K10

Key words and phrases: nonlocal diffusion-like, reaction-diffusion, uniform blow-up rate 
of overcoming this disadvantage is to introduce the non-local diffusion model related to (1.1) (see [3, 23, 24]). Equation (1.1) is called a non-local diffusion-like equation, as stated in [14], if $u(x, t)$ is thought of as the density of a species at the point $x$ and at time $t$, and $J(x-y)$ is thought of as the probability distribution of jumping from location $y$ to location $x$, then $\int_{\mathbf{R}^{N}} J(x-y) u(y, t) d y$ and $-u(x, t)=-\int_{\mathbf{R}^{N}} J(x-y) u(x, t) d y$ is the rate at which individuals are arriving at position $x$ from all other places and which they are leaving location $x$ to travel to all other sites, respectively. It is known that equation

$$
\frac{\partial}{\partial t} u(x, t)=J * u-u(x, t)=\int_{\mathbf{R}^{N}} J(x-y)(u(y, t)-u(x, t)) d y
$$

shares many properties with the classical heat equation $u_{t}=\Delta u$, such as the bounded stationary solutions and the maximum principle ([14]).

In the past few decades, many physical phenomena have been formulated into nonlocal mathematical models (see [1, 2, 8, 7, 15], [5, 6, 9], [11], [12], [14], [21] and references therein), and they have been extensively studied by many authors. In particular, Pérez-Llanos and Rossi ([21]) studied the following non-local diffusion-like equation

$$
u_{t}=\int_{\mathbf{R}^{N}} J(x-y)(u(y, t)-u(x, t)) d y+u^{p}
$$

with homogeneous Neumann boundary conditions and non-negative and non-trivial data. They concluded that the non-negative and non-trivial solutions blow up in finite time if and only if $p>1$. Moreover, they found that the blow-up rate is the same as the one that holds for the ODE $u_{t}=u^{p}$.

On the other hand, in last decades, special attentions have been paid to blow-up properties for local nonlinear degenerate or singular diffusion-like equations with local or nonlocal sources. We refer to, e.g., [28, 29, 27, 30, 25, 19, 18, 20]. It is pointed that Souplet ([27]) introduced a new method for investigating the rate and profile of blow-up of solutions of the local diffusion-like equations with nonlocal nonlinear reaction terms. For large classes of equations, he mainly proved that the solutions have global blow-up and that the rate of blowup is uniform in all compact subsets of the domain. In [19], Liu et al. discussed the following local diffusion-like equations with nonlocal nonlinear reaction terms, space integral source term or nonlocal (or localized) source and local term, that is, equations with space integral term of the form

$$
u_{t}=\Delta u+a(x) g\left(\int_{\Omega} f(u(x, t)) d x\right)
$$

equations with localized source of the form

$$
u_{t}=\Delta u+a(x) f(u(0, t))+h(u(x, t))
$$


and equations with space-time integral of the form

$$
u_{t}=\Delta u+a(x) f\left(\int_{0}^{t} \int_{\Omega} \beta(x) u(x, s) d x d s\right) .
$$

Each equation will be studied with homogeneous Dirichlet boundary conditions. They mainly determined the blow-up rate of solutions for large classes of nonlocal problems of each type above. Namely, they proved that the solutions have global blow-up, and that the blow-up rate is uniform in all compact subsets of the domain. In each case, the blow-up rate of $\|u(t)\|_{L^{\infty}(\Omega)}$ is precisely determined. However, to the best of our knowledge, there do not seem to be any results in the literature on uniform blow-up rate estimates for corresponding non-local problems of these types. In this paper, we use other techniques to prove the global blowup and to get the blow-up rate for these cases. Moreover, the blow up analysis of nonlocal dispersal equation (1.1) is different from the case of local dispersal equation (1.3). As we all know that for the local cases, we can prove the main results by invoking the regularizing effect (see e.g. [26, 29]). However, due to the lack of compactness and regularity of nonlocal dispersal operators ([10]), some difficulties, which do not arise in the study of spectral theory of local(random) dispersal operators, arise in the study of spectral theory of nonlocal dispersal operators. Hence, in order to give a complete analysis of the nonlocal problem, we must do more analysis.

In the following, we assume that

$\left(H_{1}\right) a(x)$ and $u_{0}(x)$ are radially symmetric, i.e., $a(x)=a(r)$ and $u_{0}(x)=u_{0}(r)$ with $r=|x|, a(r)$ and $u_{0}(r)$ are non-increasing for $r \in[0,1]$.

Motivated by the above works, the purpose of this paper is to analyze the blow up condition for the problem (1.1), that is, we want to show that problem (1.1) shares many important properties with the corresponding reaction diffusion equation

$$
\left\{\begin{array}{l}
u_{t}=\Delta u+a(x)\left(\int_{\Omega}|u(x, t)|^{r} d x\right)^{\frac{p}{r}}, \quad x \in \Omega, \quad t>0 \\
u(x, t)=0, \quad x \in \partial \Omega, \quad t \geq 0 \\
u(x, 0)=u_{0}(x), \quad x \in \Omega
\end{array}\right.
$$

such as blow up condition and blow up rate $([19,27])$.

As we all know that problem (1.1) can be rewritten in the form

$$
\left\{\begin{array}{l}
u_{t}=\int_{\mathbf{R}^{N}} J(x-y)(u(y, t)-u(x, t)) d y+a(x) g(t), \quad x \in \Omega, \quad t>0, \\
u(x, t)=0, \quad x \notin \Omega, \quad t \geq 0, \\
u(x, 0)=u_{0}(x), \quad x \in \Omega,
\end{array}\right.
$$

where the function $g(t) \geq 0$ depends on the solution $u$. Through this section, we use the notation $G(t)=\int_{0}^{t} g(s) d s$.

Now our main results can be stated as follows. 
THEOREM 1.1 (Blow-up). (i) Let $u$ be the solution of (1.1) and $p>1$. Then there exists $0<T<+\infty$, such that

$$
\lim _{t \rightarrow T^{-}}\|u(x, t)\|_{L^{\infty}(\Omega)}=+\infty .
$$

(ii) Let $u$ be the solution of (1.7). If there exists $0<T<+\infty$, such that $\int_{0}^{T} g(t) d t=$ $+\infty$, then

$$
\lim _{t \rightarrow T^{-}} u(x, t)=+\infty
$$

uniformly in all compact subsets of $\Omega$.

THEOREM 1.2 (Blow-up rate). Let $u(x, t)$ be the blow-up solution of (1.1) which blows up at time $T$. Moreover, assuming that $u(x, t)$ is non-decreasing in time. Then

$$
\lim _{t \rightarrow T^{-}}(T-t)^{\frac{1}{p-1}} u(x, t)=a(x)(p-1)^{\frac{1}{1-p}}\left(\int_{\Omega} a^{r}(x) d x\right)^{\frac{p}{r(1-p)}}
$$

uniformly in all compact subsets of $\Omega$.

Employing almost exactly the same arguments as in the proof of Theorem 1.2 (the minor necessary changes are left as an easy exercise to the reader), we conclude that:

COROLlary 1.1. Let $u(x, t)$ be a solution to

$$
\left\{\begin{array}{l}
u_{t}=\int_{\mathbf{R}^{N}} J(x-y)(u(y, t)-u(x, t)) d y+a(x) F(u(x, t), t), \quad x \in \Omega, \quad t>0, \\
u(x, t)=0, \quad x \notin \Omega, \quad t \geq 0 \\
u(x, 0)=u_{0}(x), \quad x \in \Omega
\end{array}\right.
$$

which blows up at time $T$.

(1) If $F=\left(\int_{0}^{t} \int_{\Omega} \beta(x) u(x, s) d x d s\right)^{p}, p>1$ and $\chi=\int_{\Omega} \beta(x) a(x) d x$, then

$$
\lim _{t \rightarrow T^{-}}(T-t)^{\frac{p+1}{p-1}} u(x, t)=a(x)(p-1)^{\frac{p+1}{1-p}}\left(\frac{2^{p}(p+1)}{\chi^{p}}\right)^{\frac{1}{p-1}}
$$

uniformly in all compact subsets of $\Omega$. Then

(2) Let $F(t)=k \exp \left(\int_{0}^{t} \int_{\Omega} \beta(x) u(x, s) d x d s\right)-1, k \geq 1$ and $\chi=\int_{\Omega} \beta(x) a(x) d x$.

$$
\lim _{t \rightarrow T^{-}}(T-t)^{\frac{p+1}{p-1}} u(x, t)=a(x)(p-1)^{\frac{p+1}{1-p}}\left(\frac{2^{p}(p+1)}{\chi^{p}}\right)^{\frac{1}{p-1}}
$$

uniformly in all compact subsets of $\Omega$.

The rest of the paper is organized as follows. In Section 2, we prove the existence and uniqueness of local solutions for problem (1.1) and show a comparison principle for the solutions. In Section 3, the blow-up rate of solutions of problem (1.1) is given. 


\section{Local existence and uniqueness of solutions to problem (1.1)}

In this section, we establish the comparison principle and some propositions about the local existence, uniqueness and boundedness of solutions to problem (1.1).

The local existence and uniqueness of the solution to problem (1.1) can be obtained via the Banach fixed point theorem.

Let $t_{0}>0$ be fixed and consider the Banach space $X_{t_{0}}=C\left(\left[0, t_{0}\right] ; C(\bar{\Omega})\right)$ with the norm

$$
\|w\|_{X_{t_{0}}}=\max _{0 \leq t \leq t_{0}}\|w(\cdot, t)\|_{L^{\infty}(\bar{\Omega})}=\max _{0 \leq t \leq t_{0}} \max _{\bar{\Omega}}|w(x, t)| .
$$

We will obtain the solution of problem (1.1) as a fixed point of the operator $F: B_{0} \rightarrow B_{0}$ defined by

$H_{w_{0}}(w)(x, t)=w_{0}(x)+\int_{0}^{t} \int_{\mathbf{R}^{N}} J(x-y)(w(y, s)-w(x, s)) d y d s+\int_{0}^{t} a(x)\left(\int_{\Omega}|w(x, s)|^{r} d x\right)^{\frac{p}{r}} d s$,

where $B_{0}=B\left(u_{0}, 2\left\|u_{0}\right\|_{L^{\infty}}\right)$. The following lemma is the main ingredient of the proof of the main results.

LEMMA 2.1. The operator $H_{u_{0}}$ is well defined, mapping $B_{0}$ into $B_{0}$. Moreover, let $w, z \in B_{0}$. Then there exists a positive constant $C=C\left(J, \Omega, u_{0}, a, p, r\right)$ such that

$$
\left\|H_{u_{0}}(w)(x, t)-H_{u_{0}}(z)(x, t)\right\|_{X_{t_{0}}} \leq C t\|w-z\|_{X_{t_{0}}} .
$$

Thus $H_{u_{0}}$ is a strict contraction in the ball $B_{0}$ provided $t_{0}$ is small enough.

PROOF. Since the convolution in space with the function $J$ being uniformly continuous, it is easy to see that $H_{u_{0}}(w)$ is continuous as the function of $x$. We first prove that the operator $H_{u_{0}}$ maps $B_{0}$ into $B_{0}$. For any $(x, t) \in \bar{\Omega} \times\left[0, t_{0}\right]$, we have

$$
\begin{aligned}
& \left|H_{u_{0}}(w)(x, t)-u_{0}(x)\right| \\
\leq & \left|\int_{0}^{t} \int_{\mathbf{R}^{N}} J(x-y)(w(y, s)-w(x, s)) d y d s+\int_{0}^{t} a(x)\left(\int_{\Omega}|w|^{r} d x\right)^{\frac{p}{r}} d s\right| \\
\leq & \int_{0}^{t} \int_{\mathbf{R}^{N}} J(x-y)|w(y, s)-w(x, s)| d y d s+\|a\|_{L^{\infty}} \int_{0}^{t}\left(\int_{\Omega}|w|^{r} d x\right)^{\frac{p}{r}} d s \\
\leq & \left(2\|J\|_{L^{\infty}}\|w\|_{X_{t_{0}}}+\|a\|_{L^{\infty}}\|w\|_{X_{t_{0}}}^{p}|\Omega|^{\frac{p}{r}}\right) \cdot t,
\end{aligned}
$$

which assures that $H_{u_{0}}(w)$ is continuous at $t=0$. And for any $w \in B_{0}$, we conclude $H_{u_{0}}(w) \in B_{0}$. Thus $H_{u_{0}}$ maps $B_{0}$ into $B_{0}$.

In addition, for any $\left(x, t_{1}\right),\left(x, t_{2}\right) \in \bar{\Omega} \times\left[0, t_{0}\right]$, taking into account that $w$ vanishes outside $\Omega$, we have

$$
\left|H_{u_{0}}(w)\left(x, t_{2}\right)-H_{u_{0}}(w)\left(x, t_{1}\right)\right|
$$




$$
\begin{aligned}
& \leq\left|\int_{t_{1}}^{t_{2}} \int_{\mathbf{R}^{N}}(w(y, s)-w(x, s)) d y d s+\int_{t_{1}}^{t_{2}} a(x)\left(\int_{\Omega}|w|^{r} d x\right)^{\frac{p}{r}} d s\right| \\
& \leq\left|\int_{t_{1}}^{t_{2}} \int_{\mathbf{R}^{N}} J(x-y)\right| w(y, s)-w(x, s)|d y d s|+\|a\|_{L^{\infty}}\left|\int_{t_{1}}^{t_{2}}\left(\int_{\Omega}|w|^{r} d x\right)^{\frac{p}{r}} d s\right| \\
& \leq\left(2\|J\|_{L^{\infty}}\|w\|_{X_{t_{0}}}+\|a\|_{L^{\infty}}\|w\|_{X_{t_{0}}}^{p}|\Omega|^{\frac{p}{r}}\right)\left|t_{2}-t_{1}\right|,
\end{aligned}
$$

which shows that $H_{u_{0}}(w)$ is continuous in time for any $t \in\left[0, t_{0}\right]$.

To prove the estimate (2.1), we recall the following $p$-laplacian type inequality, see for instance [4]. If $p>0$ and $|a|+|b|>0$ it holds that

$$
\left|a^{p}-b^{p}\right| \leq c|a-b|(|a|+|b|)^{p-1} .
$$

Then, for any $(x, t) \in \bar{\Omega} \times\left[0, t_{0}\right]$, by the Minkowski inequality, we have

$$
\begin{aligned}
& \left|H_{u_{0}}(w)(x, t)-H_{u_{0}}(z)(x, t)\right| \\
& \leq \int_{0}^{t} \int_{\mathbf{R}^{N}}|J(x-y)[w(y, s)-z(y, s)-(w(x, s)-z(x, s))]| d y d s \\
& +\|a\|_{L^{\infty}} \int_{0}^{t}\left|\|w\|_{L^{r}}^{p}-\|z\|_{L^{r}}^{p}\right| d s \\
& \leq \int_{0}^{t} \int_{\mathbf{R}^{N}}|J(x-y)[w(y, s)-z(y, s)-(w(x, s)-z(x, s))]| d y d s \\
& +c\|a\|_{L^{\infty}} \int_{0}^{t}\left(\left|\|w\|_{L^{r}}-\|z\|_{L^{r}}\right|\right)\left(\|w\|_{L^{r}}+\|z\|_{L^{r}}\right)^{p-1} d s \\
& \leq \int_{0}^{t} \int_{\mathbf{R}^{N}}|J(x-y)[w(y, s)-z(y, s)-(w(x, s)-z(x, s))]| d y d s \\
& +c\|a\|_{L^{\infty}} \int_{0}^{t}\left(\|w-z\|_{L^{r}}\right)\left(\|w\|_{L^{r}}+\|z\|_{L^{r}}\right)^{p-1} d s
\end{aligned}
$$

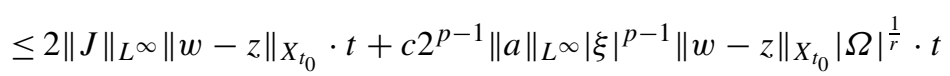

$$
\begin{aligned}
& =C t\|w-z\|_{X_{t_{0}}} \text {, }
\end{aligned}
$$

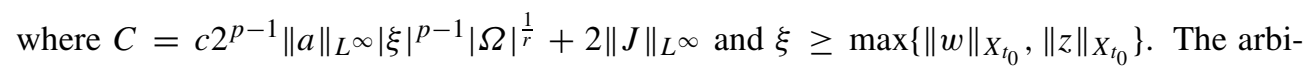
trariness of $(x, t) \in \bar{\Omega} \times\left[0, t_{0}\right]$ gives the desired estimate (2.1).

Finally, choosing $t_{0}$ such that $C t_{0}<1,(2.1)$ ensures that $H_{u_{0}}(w)$ is a strict contraction in the ball $B_{0} \subset X_{t_{0}}$ and thus the proof of this Lemma is completed.

Employing the above Lemmas, we derive:

THEOREM 2.1. For every $u_{0} \in C(\bar{\Omega})$, problem (1.1) admits a unique solution $u \in$ $C([0, T) ; C(\bar{\Omega}))$. Moreover, if the maximal existence time $T<\infty$, then the solution blows 
up in $L^{\infty}(\bar{\Omega})$-norm, that is

$$
\limsup _{t \rightarrow T}\|u(\cdot, t)\|_{L^{\infty}(\bar{\Omega})}=+\infty .
$$

PROOF. It follows from Lemma 2.1 that $F_{u_{0}}$ is a strict contraction in $B_{0}$ for $t_{0}$ small enough. By the Banach fixed point theorem, there exists only one fixed point of $F_{u_{0}}$ in $B_{0}$. This proves the existence and uniqueness of solution of $(1.1)$ in the time interval $\left[0, t_{0}\right]$. To be continue, we may take $u\left(x, t_{0}\right)$ as an initial data and obtain a unique solution of (1.1) in the time interval $\left[0, t_{1}\right]$. If $\|u\|_{X_{t_{1}}}<\infty$, taking as initial datum $u\left(\cdot, t_{1}\right) \in C(\bar{\Omega})$ and arguing as before, it is possible to extend the solution up to some interval $\left[0, t_{2}\right)$ for certain $t_{2}>t_{1}$. Hence we can conclude that if the maximal time $T$ of the existence of the solution is finite, then the solution blows up in $L^{\infty}(\bar{\Omega})$-norm, that is,

$$
\limsup _{t \rightarrow T}\|u(\cdot, t)\|_{L^{\infty}(\bar{\Omega})}=+\infty .
$$

Otherwise, the solution of problem (1.1) is global.

DEFINITION 2.1. A nonnegative function $\bar{u} \in C^{1}([0, T) ; C(\bar{\Omega}))$ is a supersolution of problem (1.1) if it satisfies

$$
\left\{\begin{aligned}
\frac{\partial}{\partial t} \bar{u}(x, t) \geq & \int_{\Omega} J(x-y)(\bar{u}(y, t)-\bar{u}(x, t)) d y \\
& -\int_{\mathbf{R}^{N} \backslash \Omega} J(x-y) \bar{u}(x, t) d y+a(x)\left(\int_{\Omega}|\bar{u}(x, t)|^{r} d x\right)^{\frac{p}{r}},(x, t) \in Q_{T} \\
\bar{u}(x, 0) \geq & u_{0}(x), \quad x \in \Omega,
\end{aligned}\right.
$$

where $Q_{T}:=\Omega \times(0, T)$. The subsolution is defined similarly by reversing the inequalities. Furthermore, if $u$ is a supersolution as well as a subsolution, then we call it a solution of problem (1.1).

LEMMA 2.2. Let $\bar{u}$ be a supersolution of problem (1.1). Then, if $u_{0} \geq 0$, we have $\bar{u}(x, t) \geq 0$ for $(x, t) \in \bar{\Omega} \times[0, T)$.

PROOF. By an approximation procedure we restrict ourselves to consider strict inequalities for the supersolution. Indeed, we can take $\bar{u}(x, t)+\delta t+\delta(\delta>0)$ as a strict supersolution, and take limit as $\delta \rightarrow 0$ at the end.

Arguing by contradiction, we assume that there exists a first time $t_{0}$ and some point $x_{0} \in \Omega$ at which $\bar{u}\left(x_{0}, t_{0}\right)=0$, and then $\bar{u}\left(y, t_{0}\right) \geq 0$ for all $y \in \Omega$. Therefore we derive

$$
\begin{aligned}
\frac{\partial}{\partial t} \bar{u}\left(x_{0}, t_{0}\right)> & \int_{\Omega} J\left(x_{0}-y\right)\left(\bar{u}\left(y, t_{0}\right)-\bar{u}\left(x_{0}, t_{0}\right)\right) d y \\
& -\int_{\mathbf{R}^{N} \backslash \Omega} J\left(x_{0}-y\right) \bar{u}\left(x_{0}, t_{0}\right) d y+a(x)\left(\int_{\Omega}\left|\bar{u}\left(y, t_{0}\right)\right|^{r} d y\right)^{\frac{p}{r}}
\end{aligned}
$$




$$
=\int_{\Omega} J\left(x_{0}-y\right) \bar{u}\left(y, t_{0}\right) d y+a(x)\left(\int_{\Omega}\left|\bar{u}\left(y, t_{0}\right)\right|^{r} d y\right)^{\frac{p}{r}} \geq 0,
$$

which contradicts with $\frac{\partial}{\partial t} \bar{u}\left(x_{0}, t_{0}\right) \leq 0$.

Applying the Lemma 2.2, we can derive:

LEMMA 2.3. Let $\bar{u}, \underline{u}$ be supersolutions and subsolutions to (1.1), respectively. Then, $\bar{u}(x, t) \geq \underline{u}(x, t)$ for every $(x, t) \in \bar{\Omega} \times[0, T)$.

\section{Blow-up condition and blow-up rate}

Once the existence and uniqueness of the solutions to problems (1.1) is ensured, we can analyze the blow-up condition for nonnegative solutions to problems (1.1).

The PRoof of Theorem 1.1. (i) For any fixed $x_{0} \in \Omega$, let $w\left(x_{0}, t\right)=g(t) \psi\left(x_{0}\right)$, where $g(t)$ satisfies the following ODE problem

$$
\left\{\begin{array}{l}
g^{\prime}(t)-\frac{a\left(x_{0}\right)\left(\int_{\Omega} \psi(x)^{r} d x\right)^{\frac{p}{r}}}{m} g^{p}(t)+\lambda_{1} g(t)=0, \quad t>0, \\
g(0)=g_{0}>0 .
\end{array}\right.
$$

Here $g_{0}$ is an appropriately big positive constant. Due to $p>1, g(t)$ is nondecreasing and there exists $0<T^{*}<+\infty$ such that $\lim _{t \rightarrow T^{*}} g(t)=+\infty$. Hence, we can infer that $w\left(x_{0}, t\right)$ is the subsolution of (1.1) provided that $g_{0} \leq \frac{\min _{x \in \bar{\Omega}} u_{0}(x)}{m}$. In fact, with the help of $p>1$, we readily find that

$$
\begin{aligned}
P w\left(x_{0}, t\right) & =w_{t}\left(x_{0}, t\right)-\left[\int_{R^{N}} J\left(x_{0}-y\right)\left(w(y, t)-w\left(x_{0}, t\right)\right) d y+a\left(x_{0}\right)\left(\int_{\Omega}\left|w^{r}\right| d x\right)^{\frac{p}{r}}\right] \\
& \leq \psi\left(x_{0}\right)\left[g^{\prime}(t)-a\left(x_{0}\right) \frac{\left(\int_{\Omega}|\psi(x)|^{r} d x\right)^{\frac{p}{r}}}{m} g^{p}(t)+\lambda_{1} g(t)\right] \\
& =0 .
\end{aligned}
$$

Here

$P w:=w_{t}(x, t)-\left[\int_{R^{N}} J(x-y)(w(y, t)-w(x, t)) d y+a(x)\left(\int_{\Omega}\left|w^{r}\right| d x\right)^{\frac{p}{r}}\right]$ and $m:=\max _{x \in \bar{\Omega}} \psi(x)$.

Thus, thanks to Lemma 2.3, we derive $u\left(x_{0}, t\right) \geq w\left(x_{0}, t\right)(0<t<T)$ for any fixed $T<T^{*}$. Therefore, $\|u(\cdot, T)\|_{L^{\infty}(\bar{\Omega})} \geq w\left(x_{0}, T\right)$, which together with the arbitrariness of $T<T^{*}$ and $\lim _{t \rightarrow T^{*}} w\left(x_{0}, t\right)=+\infty$ implies that $\lim _{t \rightarrow T^{*}}\|u(\cdot, t)\|_{L^{\infty}(\bar{\Omega})}=+\infty$. Thus, the solution of (1.1) blows up in finite time for any appropriately large initial data. 
(ii) For any $0<t<T$, integrating (1.7) 1 (the first equation of (1.7)) over $(0, t)$, we obtain

$$
\begin{aligned}
u(x, t) & =u_{0}(x)+\int_{0}^{t} \int_{\mathbf{R}^{N}} J(x-y)(u(y, s)-u(x, s)) d y d s+a(x) \int_{0}^{t} g(s) d s \\
& \geq u_{0}(x)+\int_{0}^{t} \int_{\mathbf{R}^{N}} J(x-y) u(y, s) d y d s-u(x, t) T+a(x) \int_{0}^{t} g(s) d s \\
& \geq-u(x, t) T+a(x) \int_{0}^{t} g(s) d s,
\end{aligned}
$$

that is,

$$
u(x, t) \geq a(x) \frac{\int_{0}^{t} g(s) d s}{T+1} .
$$

Now, letting $t \rightarrow T$ on both sides of (3.2) and using the fact that $\int_{0}^{T} g(t) d t=+\infty$, we can get

$$
\lim _{t \rightarrow T^{-}} u(x, t)=+\infty
$$

uniformly in all compact subsets of $\Omega$.

Next we discuss the blow-up rate, that is the speed at which the solutions blow up. Firstly, we prove a lemma that says that if the initial condition has a unique maximum at the origin, then the solution has a unique maximum at this point for every $t \in(0, T)$.

LEMMA 3.1. Under the hypothesis on the initial conditions imposed in Theorem 1.2, we have that the solution $u(x, t)$ is radially symmetric and $u_{r}<0$ in $(0,1) \times(0, T)$.

PROOF. It is easily to have that $w(x, t)=u(-x, t)$ is also a solution to (1.1). Then by the uniqueness of solution to (1.1), we have the solution $u(x, t)$ is symmetric. Let $v=u_{r}$. Then $v$ verifies the following equation

$$
v_{t}=\int_{B_{1}(0)} \sum_{i=1}^{N} \frac{\partial J\left(x_{1}-y_{1}, \ldots, x_{i}-y_{i}, \ldots, x_{N}-y_{N}\right)}{\partial x_{i}} \frac{\partial x_{i}}{\partial r} u(y, t) d y-v(x, t)+a^{\prime}(r) g(t)
$$

with $d y:=d y_{1} \ldots d y_{N}$. Notice that $J$ is a smooth and radially symmetric function, we have

$$
v_{t}=\int_{B_{1}(0)} J^{\prime}(r) u(y, t) d y-v(x, t)+a^{\prime}(r) g(t) .
$$

From this equation it is easy to obtain a contradiction, if we assume that there exists a point $\left(x_{0}, t_{0}\right) \in B_{1}(0) \times(0, T)$ at which $v\left(x_{0}, t_{0}\right)=0$. Here we have use the fact that $J^{\prime}$ is odd and the solution $u(x, t)$ is symmetric.

LEMMA 3.2. Assume $\left(H_{1}\right)$ and $g(t)$ non-negative, continuous on $(0, T)$, and $g(t)$ nondecreasing in time. Let $u$ be the blow-up solution of (1.7) and $\lim _{t \rightarrow T} G(t)=\infty$. Then we 
have

$$
\lim _{t \rightarrow T} \frac{u(x, t)}{G(t)}=a(x)
$$

uniformly in all compact subsets of $\Omega$.

Proof. Firstly, using $\lim _{t \rightarrow T} G(t)=+\infty$, we obtain

$$
\lim _{t \rightarrow T} g(t)=+\infty
$$

On the other hand, due to Lemma 3.1, we have $\max _{x \in \bar{\Omega}} u(x, t)=u(0, t)$. Therefore, by the first equation of (1.7) we have

$$
\int_{\mathbf{R}^{N}} J(x-y)(u(y, t)-u(0, t)) d y \leq 0 .
$$

Combining with (1.7) and the above inequality, we then get

$$
u_{t}(0, t) \leq a(0) g(t) \text { for all } 0<t<T,
$$

that is,

$$
\limsup _{t \rightarrow T} \frac{u(0, t)}{G(t)} \leq a(0) .
$$

Since $g(t)$ is non-decreasing and $\lim _{t \rightarrow T} G(t)=+\infty$, we have for all $\varepsilon>0$,

$$
0 \leq \frac{G(t)}{g(t)}=\frac{\int_{0}^{t} g(t) d t}{g(t)} \leq \frac{\int_{0}^{T-\varepsilon} g(t) d t}{g(t)}+\varepsilon,
$$

which together with (3.4) implies that $\lim _{t \rightarrow T} \frac{G(t)}{g(t)}=0$. Therefore, by (3.5) we derive

$$
0 \leq \lim _{t \rightarrow T} \frac{u(0, t)}{g(t)}=\lim _{t \rightarrow T} \frac{u(0, t)}{G(t)} \lim _{t \rightarrow T} \frac{G(t)}{g(t)} \leq \limsup _{t \rightarrow T} \frac{u(0, t)}{G(t)} \lim _{t \rightarrow T} \frac{G(t)}{g(t)} \leq a(0) \times 0=0,
$$

therefore,

$$
\lim _{t \rightarrow T} \frac{u(0, t)}{g(t)}=0
$$

Let $R_{1} \in(0,1), \Omega_{1}=\left\{x \in R^{N}:|x|<R_{1}\right\}$ and $b(x)=\frac{1}{a(x)}\left(x \in \Omega_{1}\right)$. Since $a^{\prime}(r) \leq 0$, we have

$$
b^{\prime}(r) \geq 0 \text { for } 0 \leq r \leq R_{1} .
$$

Now, we introduce a function

$$
w(x, t)=b(x) u(x, t), \quad x \in \Omega_{1}, \quad 0<t<T .
$$


A simple calculation yields that

$$
\begin{aligned}
& b(x) \int_{\mathbf{R}^{N}} J(x-y)(u(y, t)-u(x, t)) d y \\
= & \int_{\mathbf{R}^{N}} J(x-y)(w(y, t)-w(x, t)) d y-\int_{\mathbf{R}^{N}} J(x-y)(b(y)-b(x)) u(y, t) d y .
\end{aligned}
$$

On the other hand, by Lemma 3.1, we have

$$
u_{r} \leq 0 \text { for } 0 \leq r \leq 1 .
$$

By (3.7), (3.9) and the fact that $u(x, t)$ and $b$ are radially symmetric, we have

$$
(b(y)-b(x))(u(y, t)-u(x, t)) \leq 0 .
$$

Inserting the above inequality into (3.8), we have

$$
\begin{aligned}
& b(x) \int_{\mathbf{R}^{N}} J(x-y)(u(y, t)-u(x, t)) d y \\
\geq & \int_{\mathbf{R}^{N}} J(x-y)(w(y, t)-w(x, t)) d y-u(x, t) \int_{\mathbf{R}^{N}} J(x-y)(b(y)-b(x)) d y .
\end{aligned}
$$

Now, setting $\gamma=\max _{x \in \bar{\Omega}_{1}}\left|\int_{\mathbf{R}^{N}} J(x-y)(b(y)-b(x)) d y\right|, \rho(t)=\frac{\gamma u(0, t)}{g(t)}$. From $\lim _{t \rightarrow T} \frac{u(0, t)}{g(t)}=0$, we derive that there exists $\tau \in(0, T)$ such that $0<\rho(t) \leq \frac{1}{2}$ for $\tau \leq t<T$. Therefore, using (1.7) and (3.10), we obtain

$$
\begin{aligned}
w_{t}=b(x) u_{t} & =b(x)\left(\int_{\mathbf{R}^{N}} J(x-y)(u(y, t)-u(x, t)) d y+a(x) g(t)\right) \\
& =b(x) \int_{\mathbf{R}^{N}} J(x-y)(u(y, t)-u(x, t)) d y+g(t) \\
& \geq \int_{\mathbf{R}^{N}} J(x-y)(w(y, t)-w(x, t)) d y-u(x, t) \int_{\mathbf{R}^{N}} J(x-y)(b(y)-b(x)) d y+g(t) .
\end{aligned}
$$

Putting $g_{1}(t)=(1-\rho(t)) g(t)$ and $G_{1}(t)=\int_{\tau}^{t} g_{1}(s) d s$. We then obtain

$$
\lim _{t \rightarrow T} G_{1}(t)=+\infty
$$

and

$$
\lim _{t \rightarrow T} \frac{G_{1}(t)}{G(t)}=1 .
$$

Clearly, $w(x, t)$ is a supersolution of the following equation

$$
\left\{\begin{array}{l}
v_{t}=\int_{\mathbf{R}^{N}} J(x-y)(v(y, t)-v(x, t)) d y+g(t), \quad x \in \Omega_{1}, \quad \tau<t<T, \\
v(x, t)=0, \quad x \notin \Omega_{1}, \quad \tau<t<T \\
v(x, 0)=v_{0}(x), \quad x \in \Omega_{1}
\end{array}\right.
$$


where $0 \leq v_{0}(x) \leq w(x, \tau)$ in $\Omega_{1}$ and $v_{0}(x) \in C^{1}\left(\bar{\Omega}_{1}\right)$ with $\left.v_{0}(x)\right|_{\partial \Omega_{1}}=0$. It also assumes that $v_{0}(x)$ is symmetric and non-increasing as a function of $|x|(r=|x|)$. By the maximum principle ([14]), we have

$$
0 \leq v(x, t) \leq w(x, t) \text { and } v_{r} \leq 0 \text { in } \Omega_{1} \text { for } \tau \leq t<T .
$$

Now, let $\varphi(x)$ be the solution of

$$
\left\{\begin{array}{l}
\int_{\mathbf{R}^{N}} J(x-y)(\varphi(y)-\varphi(x)) d y=-\mu \varphi(x) \quad x \in \Omega_{1}, \\
\varphi(x)=0 \quad x \notin \bar{\Omega}_{1} .
\end{array}\right.
$$

By Theorem 2.1 of [16], problem (3.14) admits a unique eigenvalue $\mu_{1}:=\mu_{1}(\Omega)$ associated to a positive eigenfunction $\varphi_{1} \in C\left(\bar{\Omega}_{1}\right)$ which is normalized by

$$
\int_{\Omega_{1}} a(x) \varphi_{1}(x) d x=1 .
$$

Next, multiplying both sides of (3.13) 1 by $\varphi_{1}$ and integrating over $\Omega_{1} \times(\tau, t)$, we have

$$
\begin{aligned}
& \int_{\Omega_{1}} v(x, t) \varphi_{1}(x) d x-\int_{\Omega_{1}} v_{0}(x) \varphi_{1}(x) d x \\
& \quad=\mu_{1} \int_{\tau}^{t} \int_{\Omega_{1}} v(x, s) \varphi_{1}(x) d x d s+G_{1}(t) \text { for } \tau<t<T .
\end{aligned}
$$

By (3.6) and (3.15) $v(0, s)=\max _{x \in \bar{\Omega}_{1}} v(x, s)$ (for $\tau \leq s<t$ ), we have

$$
\int_{\tau}^{t} \int_{\Omega_{1}} v(x, s) \varphi_{1}(x) d x d s \leq \int_{\tau}^{t} v(0, s) d s \leq \int_{\tau}^{t} w(0, s) d s
$$

and

$$
\lim _{t \rightarrow T} \frac{w(0, t)}{g_{1}(t)}=\lim _{t \rightarrow T} \frac{w(0, t)}{g(t)}=\frac{1}{a(0)} \lim _{t \rightarrow T} \frac{u(0, t)}{g(t)}=0,
$$

which together with (3.12) implies

$$
\lim _{t \rightarrow T} \frac{\int_{\tau}^{t} \int_{\Omega_{1}} v(x, s) \varphi_{1}(x) d x d s}{G_{1}(t)}=\lim _{t \rightarrow T} \frac{\int_{\tau}^{t} \int_{\Omega_{1}} v(x, s) \varphi_{1}(x) d x d s}{G(t)}=0 .
$$

Inserting the above inequality into (3.16) and applying the basic calculation, we have

$$
\lim _{t \rightarrow T} \frac{\int_{\Omega_{1}} v(x, t) \varphi_{1}(x) d x}{G_{1}(t)}=1,
$$

which implies that

$$
\lim _{t \rightarrow T} \inf \frac{v(0, t)}{G_{1}(t)} \geq 1 .
$$


On the other hand, by (3.13) and $\int_{\mathbf{R}^{N}} J(x-y)(v(y, t)-v(0, t)) d y \leq 0$, we have

$$
v_{t}(0, t) \leq g_{1}(t), \quad \tau<t<T,
$$

Integrating the above inequality over $(0, t)$,

$$
v(0, t)-v(0,0) \leq G_{1}(t),
$$

that is,

$$
\lim _{t \rightarrow T} \sup \frac{v(0, t)}{G_{1}(t)} \leq 1
$$

which together with (3.18) implies that

$$
\lim _{t \rightarrow T} \frac{v(0, t)}{G_{1}(t)}=1 .
$$

Applying $\int_{\Omega_{1}} \varphi_{1}(x) d x=1, v_{r} \leq 0,(3.17)$ and (3.20), we derive

$$
\lim _{t \rightarrow T} \frac{v(x, t)}{G_{1}(t)}=\lim _{t \rightarrow T} \frac{v(x, t)}{G(t)}=1
$$

uniformly in all compact subsets of $\Omega_{1}$, that is,

$$
\lim _{t \rightarrow T} \inf \frac{u(x, t)}{G(t)} \geq a(x) \text { uniformly in all compact subsets of } \Omega_{1} .
$$

Due to the arbitrariness of $\Omega_{1}$, we obtain

$$
\lim _{t \rightarrow T} \inf \frac{u(x, t)}{G(t)} \geq a(x) \text { uniformly in all compact subsets of } \Omega \text {. }
$$

In particular,

$$
\lim _{t \rightarrow T} \inf \frac{u(0, t)}{G(t)} \geq a(0)
$$

which combined with (3.5) implies that

$$
\lim _{t \rightarrow T} \inf \frac{u(0, t)}{G(t)}=a(0) .
$$

Now, multiplying (1.7) $)_{1}$ by $\varphi$ and integrating over $\Omega \times(0, t)$, we have,

$$
\begin{aligned}
\int_{\Omega} u & (x, t) \varphi(x) d x-\int_{\Omega} u_{0}(x) \varphi(x) d x \\
& =\mu_{1} \int_{0}^{t} \int_{\Omega} u(x, s) \varphi(x) d x d s+G(t) \text { for } 0<t<T .
\end{aligned}
$$


Due to $\int_{0}^{t} \int_{\Omega} u(x, s) \varphi(x) d x d s \leq \int_{\Omega} \varphi(x) d x \int_{0}^{t} u(0, s) d s$ and $\lim _{t \rightarrow T} \frac{u(0, t)}{g(t)}=0$, we have

$$
\lim _{t \rightarrow T} \frac{\int_{0}^{t} \int_{\Omega} u(x, s) \varphi(x) d x d s}{G(t)}=0,
$$

which together with (3.26) implies that

$$
\lim _{t \rightarrow T} \frac{\int_{\Omega} u(x, t) \varphi(x) d x}{G(t)}=1 .
$$

In the following, we will prove that

$$
\lim _{t \rightarrow T} \frac{u(x, t)}{G(t)}=a(x) \text { uniformly in all compact subsets of } \Omega \text {. }
$$

Assuming that there exists $x_{0} \in \Omega \backslash 0$ such that $\lim _{t \rightarrow T} \frac{u(x, t)}{G(t)}=c>a\left(x_{0}\right)$, that is, there exists a sequence $t_{n} \rightarrow T$ such that $\lim _{t_{n} \rightarrow T} \frac{u\left(x, t_{n}\right)}{G\left(t_{n}\right)}=c$. Using the continuity of $a(x)$, we deduce that there exists $x_{1} \in \Omega\left(\left|x_{1}\right|<\left|x_{0}\right|\right)$ such that $a(x)<c$ for $\left|x_{1}\right| \leq|x| \leq\left|x_{0}\right|$. By $u_{r} \leq 0$, (3.25) and (3.28), we deduce that

$$
\begin{aligned}
\lim _{t \rightarrow T} & \int_{\Omega} \frac{u(x, t) \varphi(x)}{G(t)} d x \\
& =\lim _{t_{n} \rightarrow T}\left(\int_{\left|x_{0}\right|<|x|<R} \frac{u\left(x, t_{n}\right) \varphi(x)}{G\left(t_{n}\right)} d x+\int_{\left|x_{1}\right|<|x|<\left|x_{0}\right|} \frac{u\left(x, t_{n}\right) \varphi(x)}{G\left(t_{n}\right)} d x+\int_{|x|<\left|x_{1}\right|} \frac{u\left(x, t_{n}\right) \varphi(x)}{G\left(t_{n}\right)} d x\right) \\
& \geq \int_{\left|x_{0}\right|<|x|<R} a(x) \varphi(x) d x+\lim _{t_{n} \rightarrow T} \int_{\left|x_{1}\right|<|x|<\left|x_{0}\right|} \frac{u\left(x, t_{n}\right) \varphi(x)}{G\left(t_{n}\right)} d x+\int_{|x|<\left|x_{1}\right|} a(x) \varphi(x) d x \\
& =\int_{\left|x_{0}\right|<|x|<R} a(x) \varphi(x) d x+c \int_{\left|x_{1}\right|<|x|<\left|x_{0}\right|} \varphi(x) d x+\int_{|x|<\left|x_{1}\right|} a(x) \varphi(x) d x \\
& >\int_{\left|x_{0}\right|<|x|<R} a(x) \varphi(x) d x+\int_{\left|x_{1}\right|<|x|<\left|x_{0}\right|} a(x) \varphi(x) d x+\int_{|x|<\left|x_{1}\right|} a(x) \varphi(x) d x=1 .
\end{aligned}
$$

This contradicts (3.28) and we then get the desired result.

The PRoOF OF THEOREM 1.2. Set $g(t)=\|u(t)\|_{L^{r}(\Omega)}^{p}, G(t)=\int_{0}^{t} g(s) d s$. Since $u(x, t)$ is non-decreasing in time, it then follows by $\left(H_{1}\right)$ that $g^{\prime}(t) \geq 0(t>0)$ and $u_{r} \leq 0$ on $[0,1]$ for $t>0$ that

$$
\lim _{t \rightarrow T} u(0, t)=\infty .
$$

Obviously,

$$
u_{t}(0, t) \leq a(0) g(t), 0<t<T,
$$


which implies

$$
\lim _{t \rightarrow T} \sup \frac{u(0, t)}{G(t)} \leq a(0),
$$

which combined with (3.31) implies that

$$
\lim _{t \rightarrow T} G(t)=\infty
$$

Hence, by Theorem 3.2, we have

$$
\lim _{t \rightarrow T} \frac{u(x, t)}{G(t)}=a(x) \text { uniformly in all compact subsets of } \Omega .
$$

\section{References}

[1] A. Z. AkCASU and E. DAniels, Fluctuation analysis in simple fluids, Phys. Rev. A 2 (1970), 962.

[2] W. E. Alley and B. J. Alder, Generalized transport coefficients for hard spheres, Phys. Rev. A 27 (1983), 3158.

[ 3 ] F. Andreu, J. M. Mazón, J. D. Rossi and J. Toledo, Non-Local Diffusion Problems, Mathematical Surveys and Monographs, vol. 165, 2010.

[ 4 ] J. W. BARRETT and W. B. LiU, Finite element approximation of the parabolic $p$-Laplacian, SIAM J. Numer. Anal. 31 (1994), 413-428.

[ 5 ] P. BATES and A. ChMAJ, An integrodifferential model for phase transitions: stationary solutions in higher dimensions, J. Statistical Phys. 95 (1999), 1119-1139.

[6] P. BATES, P. FIFE, X. REN and X. WANG, Travelling waves in a convolution model for phase transitions, Arch. Rat. Mech. Anal. 138 (1997), 105-136.

[ 7 ] J. Boon and S. YIP, Molecular Hydrodynamics, McGraw-Hill, New York, 1980.

[ 8 ] P. J. Cadusch, B. D. Todd, J. Zhang and P. J. Daivis, A non-local hydrodynamic model for the shear viscosity of confined fluids: analysis of a homogeneous kernel, J. Phys. A 41(3) (2008), 035501. 23 pp.

[9] C. Carrillo and P. Fife, Spatial effects in discrete generation population models, J. Math. Biol. 50(2) (2005), 161-188.

[10] E. Chasseigne, M. Chaves and J. D. Rossi, Asymptotic behaviour for nonlocal diffusion equations, J. Math. Pures Appl. 86 (2006), 271-291.

[11] X. CHEN, Existence, uniqueness and asymptotic stability of travelling waves in nonlocal evolution equations, Adv. Diff. Eqns. 2 (1997), 125-160.

[12] J. Coville, J. DÁila and S. Martinez, Nonlocal anisotropic dispersal with monostable nonlinearity, J. Diff. Eqns. 244 (2008), 3080-3118.

[13] R. Ferreira and M. PÉREZ-Llanos, Blow-up for the non-local $p$-Laplacian equation with a reaction term, Nonlinear Anal. TMA. 75 (2012), 5499-5522.

[14] P. FIfE, Some Nonclassical Trends in Parabolic and Parabolic-like Evolutions, Trends in nonlinear analysis, 153-191, Springer, Berlin, 2003.

[15] J. García-Mellán and F. Quirós, Fujita exponents for evolution problems with nonlocal diffusion, J. Evol. Eqns. 10 (2010), 147-161.

[16] J. García-Melián and J. D. Rossi, On the principal eigenvalue of some nonlocal diffusion problems, J. Diff. Eqns. 246 (2009), 21-38.

[17] L. Hopf, Introduction to Differential Equations of Physics, Dover, New York, 8 (1948), 55-100.

[18] Q. LIU, Y. LI and H. GAO, Uniform blow-up rate for a nonlocal degenerate parabolic equations, Nonlinear Anal. TMA. 66(4) (2007), 881-889. 
[19] Q. LIU, Y. LI and H. GAO, Uniform blow-up rate for diffusion equations with nonlocal nonlinear source, Nonlinear Anal. TMA. 67(6) (2007), 1947-1957.

[20] Q. LIU, Y. LI and H. GAO, Uniform blow-up rate for diffusion equations with localized nonlinear source, J. Math. Anal. Appl. 320(2) (2006), 771-778.

[21] M. PÉrez-Llanos and J. D. Rossi, Blow-up for a non-local diffusion problem with Neumann boundary conditions and a reaction term, Nonlinear Anal. TMA. 70 (2009), 1629-1640.

[22] P. Morse and H. Feshback, Methods of Theoretical Physics, McGraw Hill, New York, 1 (1953).

[23] J. D. Murray, Mathematical Biology, Springer, New York, 1993.

[24] S. PAN, W. LI and G. LIN, Travelling wave fronts in nonlocal delayed reaction-diffusion systems and applications, Z. Angew. Math. Phys. 60 (2009), 377-392.

[25] C. QU, X. BAI and S. ZHENG, Blow-up versus extinction in a nonlocal $p$-Laplace equation with Neumann boundary conditions, J. Math. Anal. Appl. 412(1) (2014), 326-333.

[26] P. Souplet, Uniform blow-up profle and boundary behaviour for a non-local reaction-diffusion equation with critical damping, Math. Meth. Appl. Sci. 27 (2004), 1819-1829.

[27] P. SOUPLET, Uniform blow-up profiles and boundary behavior for diffusion equations with nonlocal nonlinear source, J. Diff. Eqns. 153 (1999), 374-406.

[28] P. Souplet, Blow-up in nonlocal reaction-diffusion equations, SIAM J. Math. Anal. 29 (1998), 1301-1334.

[29] M. WANG and Y. WANG, Properties of positive solutions for non-local reaction-diffusion problems, Math. Method. Appl. Sci. 19 (1996), 1141-1156.

[30] C. WANG, S. ZHENG and Z. WANG, Critical Fujita exponents for a class of quasilinear equations with homogeneous Neumann boundary data, Nonlinearity 20(6) (2007), 1343-1359.

Present Address:

SCHOOL OF MATHEMATICS AND STATISTICS SCIENCE,

LUDONG UNIVERSITY,

YANTAI 264025, P.R.CHINA.

e-mail: zhengjiashan2008@163.com 